\title{
First evidence of pulsations in Vega?^
}

\section{Results of today's most extensive spectroscopic search}

\author{
T. Böhm ${ }^{1,2}$, F. Lignières ${ }^{1,2}$, G. Wade ${ }^{4}$, P. Petit ${ }^{1,2}$, M. Aurière ${ }^{1,2}$, W. Zima ${ }^{3}$, A. Fumel ${ }^{1,2}$, and E. Alecian ${ }^{5}$ \\ 1 Université de Toulouse; UPS-OMP; IRAP; Toulouse, France \\ e-mail: boehm@obs-mip.fr \\ 2 CNRS; IRAP; 14 avenue Edouard Belin, 31400 Toulouse, France \\ 3 Instituut voor Sterrenkunde, Celestijnenlaan 200D BUS 2401, Belgium \\ ${ }^{4}$ Dept. of Physics, Royal Military College of Canada, PO Box 17000, Stn Forces, Kingston K7KK 7B4, Canada \\ 5 Observatoire de Paris-Meudon, LESIA, 92190 Meudon, France
}

Received 27 September 2011 / Accepted 7 December 2011

\begin{abstract}
Context. The impact of rapid rotation on stellar evolution theory remains poorly understood as of today. Vega is a special object in this context as spectroscopic and interferometric studies have shown that it is a rapid rotator seen nearly pole one, a rare orientation particularly interesting for seismic studies. In this paper, we present a first systematic search for pulsations in Vega.

Aims. The goal of the present work is to detect for the first time pulsations in a rapidly rotating star seen nearly pole-on.

Methods. Vega was monitored in quasi-continuous high-resolution echelle spectroscopy. A total of 4478 spectra were obtained. More precisely in 2008 we obtained 1213 spectra during $19.9 \mathrm{~h}$ on 3 nights (26th, 27th and 29th of July 2008) with NARVAL/TBL (at $R=65000$ and $R=75000$ ), in 2009 we obtained 1293 spectra during $13.7 \mathrm{~h}$ on 3 nights (9th-11th of September 2009) with ESPaDOnS/CFHT (at $R=68000$ ) and in 2010 we gathered again 1972 with NARVAL/TBL during 28.8 h on five nights (July 15 th19th). This data set should represent the most extensive high $S / N$, high resolution quasi-continuous survey obtained on Vega as of today. Least square deconvolved (LSD) profiles were obtained for each spectrum representing the photospheric absorption profile potentially deformed by the presence of pulsations. In addition, we calculated for each spectrum a telluric line LSD profile subsequently used as radial velocity reference. LSD profile centroids were adjusted and velocity differences (stellar-telluric) determined. These residual velocities were analysed and periodic low amplitude variations, potentially indicative of stellar pulsations, detected. In a subsequent step, the temporal line profile variations during the longest (2010) data set was calculated for each individual velocity bin of $1.8 \mathrm{~km} \mathrm{~s}^{-1}$ by computing a 2D (velocity-frequency) Lomb-Scargle periodogram.

Results. Based on high resolution echelle spectroscopy, we have obtained indications of periodic variations of very small amplitudes within the residual radial velocity curves of Vega. All three data sets revealed the presence of residual periodic variations: 5.32 and $9.19 \mathrm{~d}^{-1}\left(A \approx 6 \mathrm{~m} \mathrm{~s}^{-1}\right)$ in $2008,12.71$ and $13.25 \mathrm{~d}^{-1}\left(A \approx 8 \mathrm{~m} \mathrm{~s}^{-1}\right)$ in 2009 and 5.42 and $10.82 \mathrm{~d}^{-1}\left(A \approx 3-4 \mathrm{~m} \mathrm{~s}^{-1}\right)$ in 2010. However, it is too early to conclude that the variations are due to stellar pulsations, and a confirmation of the detection with a highly stable spectrograph is a necessary next step.

Conclusions. If pulsations are confirmed, their very small amplitudes show that the star would belong to a category of very "quiet" pulsators.
\end{abstract}

Key words. stars: oscillations - stars: rotation - stars: individual: Vega - asteroseismology

\section{Introduction}

Rapid rotation is a characteristic feature of massive and intermediate mass stars that has a significant and yet poorly understood impact on their structure and evolution. It is a current challenge of stellar physics to obtain observational constraints that will improve the modeling of the physical processes induced by rotation. Seismology should play a particular role in this context as it is the only way to directly probe stellar interiors.

At present, however, the oscillation spectra of rapidly rotating stars can not be unambiguously interpreted. One difficulty

* Based on observations obtained at the Bernard Lyot Telescope (TBL, Pic du Midi, France) of the Midi-Pyrénées Observatory, which is operated by the Institut National des Sciences de l'Univers (INSU) of the Centre National de la Recherche Scientifique of France (CNRS), and at the Canada-France-Hawaii Telescope (CFHT), which is operated by the National Research Council of Canada, INSU/CNRS and the University of Hawaii. resides in the modeling of the effects of rapid rotation on the oscillation modes. There has been some recent progress in this field notably in the description of the organization of p-modes spectra (Lignières \& Georgeot 2009). Even if we understand the basic properties of the frequency spectrum, the mode identification process remains a difficult task. In particular, in the p-mode frequency range, the large rotational splittings between modes of different azimuthal number mix the frequencies of different multiplets. One possible solution to this problem would be to observe a rapidly rotating pulsating star seen pole-on. Cancellation effect in disk-integrated light would then select axisymmetric modes, non-axisymmetric modes being cancelled out. Such a selection effect would considerably simplify the observed spectrum and thus the mode identification process.

Vega is known to be a rapidly rotating star and reveals a nearly pole on inclination. This has been shown by analyzing the spectroscopic and interferometric signatures of the surface gravity darkening (Gulliver et al. 1994; Peterson et al. 2006). 
Table 1. Log of the spectroscopic (and polarimetric) observations of Vega.

\begin{tabular}{lcccccc}
\hline \hline $\begin{array}{l}\text { Date } \\
(1)\end{array}$ & $\begin{array}{c}\mathrm{JD}_{\text {first }} \\
(2)\end{array}$ & $\begin{array}{c}\mathrm{JD}_{\text {last }} \\
(3)\end{array}$ & $\begin{array}{c}N_{\text {spec }} \\
(4)\end{array}$ & $\begin{array}{c}t_{\text {exp }}(\mathrm{s}) \\
(5)\end{array}$ & $\begin{array}{c}t_{\text {cov }}(\mathrm{h}) \\
(6)\end{array}$ & $\begin{array}{c}S / N \\
(7)\end{array}$ \\
\hline Jul. 26 2008 & 4674.3489 & 4674.6072 & 389 & 6 & 6.2 & $812 \pm 50$ \\
Jul. 27 2008 & 4675.3748 & 4675.6551 & 390 & 6 & 6.7 & $822 \pm 61$ \\
Jul. 29 2008 & 4677.3480 & 4677.6415 & 434 & 6 & 7.0 & $823 \pm 82$ \\
\hline Sep. 9 2009 & 5083.7400 & 5083.9066 & 402 & 4 & 4.0 & $865 \pm 85$ \\
Sep. 10 2009 & 5084.7111 & 5084.8787 & 331 & 4 & 4.0 & $887 \pm 69$ \\
Sep. 11 2009 & 5085.7122 & 5085.9498 & 560 & 4 & 5.7 & $736 \pm 155$ \\
\hline Jul. 15 2010 & 5393.3643 & 5393.5735 & 355 & 13 & 5.0 & $1117 \pm 37$ \\
Jul. 16 2010 & 5394.3635 & 5394.6576 & 496 & 13 & 7.1 & $1051 \pm 158$ \\
Jul. 17 2010 & 5395.3650 & 5395.6510 & 453 & 13 & 6.9 & $1197 \pm 59$ \\
Jul. 18 2010 & 5396.3580 & 5396.4662 & 185 & 13 & 2.6 & $1222 \pm 16$ \\
Jul. 19 2010 & 5397.3561 & 5397.6569 & 483 & 13 & 7.2 & $1170 \pm 70$ \\
\hline
\end{tabular}

Notes. (1) Date of the observation (UT) (Petit et al. (2010) indicates the date in local time, leading to a -1 day shift for the Sept. 2009 CFHT data). (2) and (3) heliocentric Julian date (mean observation, $2450000+$ ) of the first and the last stellar spectrum of the night, respectively; (4) number of high resolution RS Cha spectra; (5) exposure time (s); (6) total hours covered on the sky; (7) nightly average and standard deviation of signal to noise per resolved element at $520 \mathrm{~nm}$.

The spectroscopic and interferometric approaches provide a similar inclination angle $i=5-7$ degrees, although they differ in the reported equatorial velocities, $v_{\mathrm{eq}}=245 \pm 15 \mathrm{~km} \mathrm{~s}^{-1}$, $v_{\text {eq }}=211 \pm 4 \mathrm{~km} \mathrm{~s}^{-1}$ and $v_{\text {eq }} \sim 175 \mathrm{~km} \mathrm{~s}^{-1}$ from the spectroscopic analysis of Gulliver et al. (1994), Hill et al. (2010) and Takeda et al. (2008), respectively, and a significantly larger value, $v_{\text {eq }} \sim 275 \mathrm{~km} \mathrm{~s}^{-1}$, from interferometry (Peterson et al. 2006; Aufdenberg et al. 2006). As a seismic target, Vega also presents the important advantage that its fundamental parameters are very accurately determined, particularly when compared with other rapidly rotating stars.

As reviewed by Gray (2007), Vega was used since more than 150 years as a photometric and spectrophotometric standard star, the historic reason being most likely its brightness and nearzenith position for european and north american observatories. This particular role of Vega can be considered surprising, since several authors report photometric variability of at least $1-2 \%$, but with no evidence of periodic variations. A previous study of spectroscopic variability has been conducted by Goraya \& Singh (1983) who described a variability of $\mathrm{H} \alpha$ althought it was not confirmed in subsequent studies (Charlton \& Meyer 1985). Very small amplitude variations of a few thousandths of the continuum are reported for some of the weakest lines on a time basis of several years (Hill et al. 2010).

The location of Vega in the HR diagram is roughly that of main-sequence A0 stars, but bluewards of the $\delta$ Scuti instability strip, therefore stellar oscillations are in principle not expected. However, Fernie (1981) concluded that historic radial velocity curves and photometric variability tend to indicate episodical $\delta$ Scuti type pulsation, with a frequency close to the fundamental radial order of $F_{\text {fund }} \sim 10 \mathrm{~d}^{-1}$ for Vega's parameters as known at that time. The most recent determination of fundamental stellar parameters by Takeda et al. (2008) led him to conclude that this rapid rotator has parameters strongly varying from pole to equator, more precisely an effective temperature from $T_{\text {eff }}=9867$ to $8931 \mathrm{~K}$, a surface gravity from $\log g=3.308$ to 3.313 (where $g$ is expressed in $\mathrm{cm} \mathrm{s}^{-2}$ ) and a radius from $R=2.520$ to $2.763 R_{\odot}$, respectively.

Until know, no rapidly rotating, pole-on and observably pulsating star was known. The goal of our work was therefore to search for low amplitude stellar pulsations in an extensive highresolution quasi-continuous spectroscopic survey of Vega.
Section 2 describes the observations and data reduction, Sect. 3 presents the analysis of radial velocity variations in the spectroscopic time series of Vega, and in Sect. 4 results are discussed and a conclusion is drawn.

\section{Observations and data reduction}

The analysis presented in this paper is based on three datasets obtained in 2008, 2009 and 2010. Vega was monitored during 3 nights in 2008 (26th, 27th and 29th of July) with the high-resolution echelle spectropolarimeter NARVAL at the $2 \mathrm{~m}$ Bernard Lyot Telescope (TBL, Pic du Midi, France). The first two nights observations were acquired at $R=65000$ in polarimetric mode, and the last night at $R=75000$ in spectroscopic mode. We obtained 1213 spectra covering 19.9 h. In 2009 we obtained 1293 spectra covering $13.7 \mathrm{~h}$ on 3 nights (9th-11th of September 2009) at ESPaDOnS/CFHT $(R=68000)$ in polarimetric mode. Finally, in 2010, we got a huge data set of 1972 spectra covering 28.8 h on 5 nights (15th-19th of July 2010), obtained again in the polarimetric mode at NARVAL/TBL. Table 1 summarizes the log of the observations during these 3 runs.

The general observing strategy was to obtain as many maximum $S$ / $N$ observations of the target during the night as possible. The goal was to obtain a versatile data set, enabling us to search for the presence of a magnetic field as well as stellar pulsations. Results of the spectropolarimetric study of the first observing run are presented in Lignières et al. (2009), where the detection of a weak magnetic field in Vega has been announced, revealing the existence of an as-yet unexplored class of weakly magnetic stars in the intermediate mass domain. The joint analysis of the two first runs (2008 and 2009) confirmed this first detection and indicated a rotational modulation of the polarization signature with a period of $0.732 \pm 0.008 \mathrm{~d}$, a value which is very close to the rotation period announced by Takeda et al. $(2008)(\simeq 0.733 \mathrm{~d})$. These results are published in Petit et al. (2010). A recent analysis of the 2010 data set yielded a slightly different rotation period of $0.678_{-0.029}^{+0.036}$ d (Alina et al. 2011).

The global data set containing 4478 spectra obtained at the highest possible signal to noise should represent, as of today, the most extensive high resolution quasi-continuous spectroscopic monitoring of Vega. It should be noted that for such a bright star the exposure times are extremely short ( 4 to $13 \mathrm{~s}$ on the star) in order to avoid the saturation of the CCD, while the readout 
Photospheric lines in Vega's spectrum - 18th July 2010

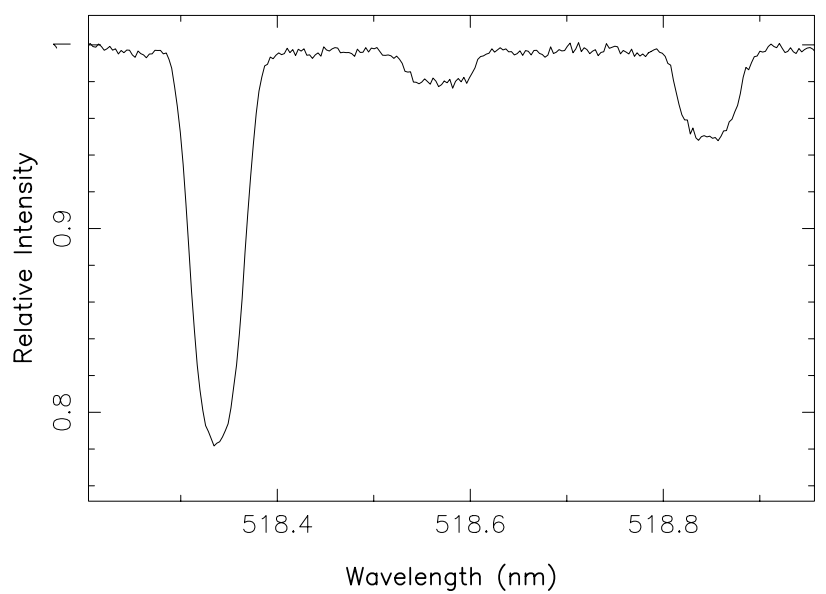

Fig. 1. Photospheric line profiles in Vega's spectrum. Strong lines exhibit classical, rotationally broadened line profiles, while weak lines show flat-bottomed trapezoidal profiles indicative of gravity darkening. The three dominant lines in this figure are: Mg I 5183.60 ̊, Ti II $5185.90 \AA$ A (very slightly blended with a Si II line) and Ti II 5188.68 Å.

times of the CCD dominate the duty-cycle, the average time interval between two exposures being close to $50 \mathrm{~s}$. Subexposures obtained in polarimetric mode provide as a direct result from the data reduction pipeline, in addition to the polarization information, a well calibrated intensity spectrum.

The wavelength covered extends from 369 to $1048 \mathrm{~nm}$ for both spectrographs. Standard calibration spectra were obtained at the beginning and at the end of the night; most importantly the absolute wavelength calibration relies on a Th/Ar spectrum at the beginning of the night. Most of the data reduction was carried out following standard reduction procedures using a dedicated spectroscopic data reduction package, based on the algorithm described in Donati et al. (1997). This package also makes use of the "optimal extraction algorithm" (Horne 1986). A standard heliocentric velocity correction was performed. The intrinsic wavelength calibration accuracy achieved with the 2D-polynomial fit procedure is better than $150 \mathrm{~m} \mathrm{~s}^{-1}$ for both data sets.

Figure 1 shows photospheric spectral lines of different intensities present in Vega's spectrum, obtained on July 18th 2010 at TBL. Figure 2 shows a section of the same spectrum containing lines studied by Takeda et al. (2008), revealing the particular flat-bottomed trapezoidal profile indicative of the action of strong gravity darkening, as discussed later in this section. It can be clearly seen on these two figures that the spectrum of Vega is much more complex than expected from an A0 standard star, despite the fact that the synthetic spectrum represented in our figure does not exactly match Vega's surface gravity.

In order to be able to calibrate potential spectral shifts during the night a cross-correlation of telluric lines present in the spectra was performed by the standard reduction package. After all shifts have been applied to the wavelength grid, the residual telluric line velocity should reflect precisely the opposite of the heliocentric velocity correction. In order to verify this, we computed least square deconvolved (LSD) profiles (Donati et al. 1997) of the more than 100 telluric water vapor lines contained in each stellar spectrum, and determined their centroid by fitting a gaussian profile. Figure 3 shows some of the deepest telluric lines present in Vega's spectrum, obtained on July 18th 2010 at TBL. We discovered the presence of residual radial velocity errors of the telluric lines. In order to optimize the radial velocity

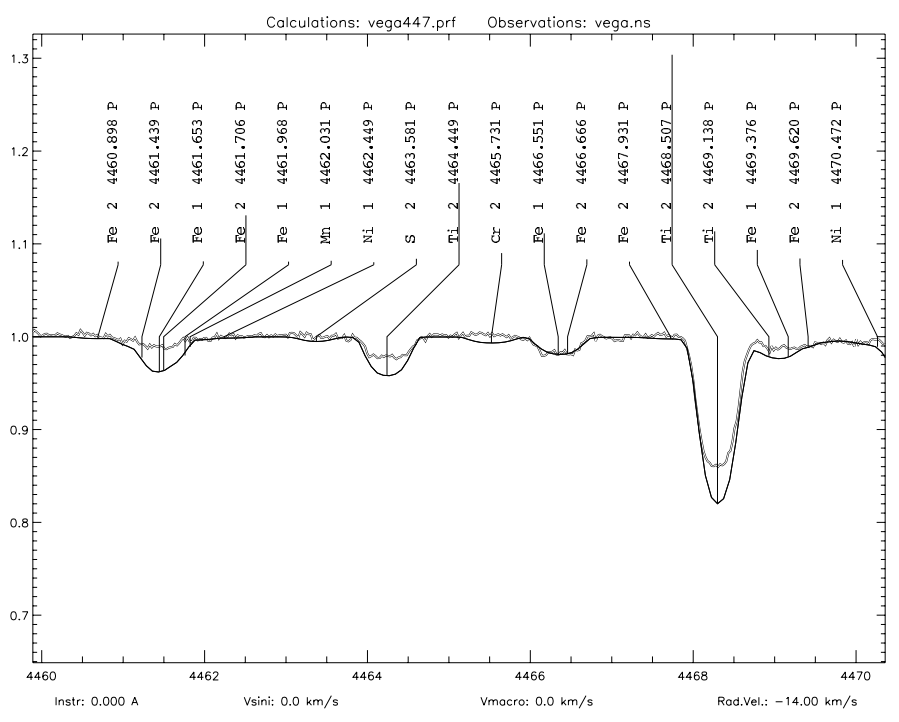

Fig. 2. Flat-bottomed photospheric line profiles in Vega's spectrum indicative of strong gravity darkening. Superimposed is a synthetic spectrum with $T_{\text {eff }}=10000 \mathrm{~K}, \log g=3.5, v \sin i=22 \mathrm{~km} \mathrm{~s}^{-1}$ and $v_{\text {rad }}=$ $-14.0 \mathrm{~km} \mathrm{~s}^{-1}$ calculated with SYNTH3 (Kochukhov 2007).

Telluric lines in Vega's spectrum - 18th July 2010

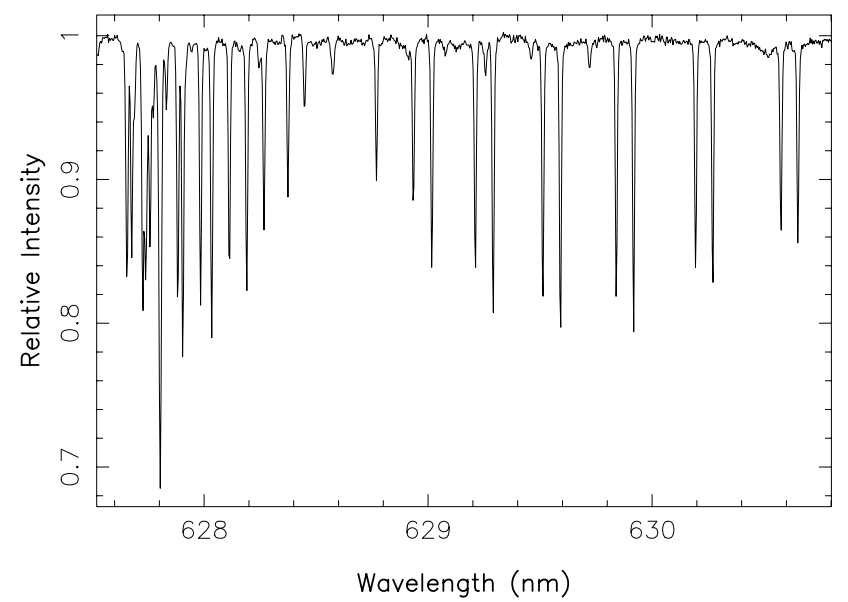

Fig. 3. Example of telluric lines present in Vega's spectrum.

precision we corrected then for the remaining velocity shifts. The residual spectral stability is around $10-15 \mathrm{~m} \mathrm{~s}^{-1}$ (depending on the night and on the site), taking into account all the multiplex information of the lines present in the spectra.

At this point it should be noted that a stability analysis of $\mathrm{O}_{2}$ telluric lines has been carried out by Caccin et al. (1985). They report that the line positions change more or less erratically with time, their displacement depending upon the horizontal wind structure above the observing site. They note however, that unless the observations are made near the horizon, the component of the wind velocity along the line of sight is very seldom significant, the displacement reaching up to $10 \mathrm{~m} \mathrm{~s}^{-1}$ only in very severe conditions (large air mass, strong winds). In low wind conditions and for only one to three airmasses their time instability should be probably of the order of $5 \mathrm{~m} \mathrm{~s}^{-1}$. This is the case for our observations of Vega, since they have been obtained in airmasses between 1 and 2 , and only during the last night of the CFHT run airmasses up to 2.6 were reached. In a recent work, Figueira et al. (2010) evaluated the stability of atmospheric lines with HARPS (on the $3.6 \mathrm{~m}$ telescope at La Silla, 
ESO), and concluded in a similar way to Caccin et al. (1985) that a precision of $5 \mathrm{~m} \mathrm{~s}^{-1}$ can routinely be obtained in restricted airmass conditions. What we retain from this latter work is the fact that increasing airmass induces asymmetry of telluric lines, this asymmetry is therefore expected to have an impact on the measured RV. This dependence on the airmass of the observed object repeats therefore every night for the same target (within a 3 or 5 night observing run). Any periodic behaviour with different periodicities than 1d cannot be attributed to this intrinsic telluric line variability. Moreover, even if Caccin et al. (1985) invoke high altitude atmospheric movements or pressure shifts as responsible for inducing asymmetry in telluric lines, we could not find any report of sinusoidal radial velocity variation of telluric lines during an observing night.

The next step of the data reduction was to calculate for all stellar spectra photospheric LSD profiles, using a mask corresponding to the spectral type A0 (see also Petit et al. 2010). We have to notice that, as Gulliver et al. (1994) and Takeda et al. (2008) have shown, the spectrum of this rapidly rotating star is very inhomogeneous, since the weak spectral lines form exclusively in the almost $1000 \mathrm{~K}$ cooler gravity darkened equatorial zone, leading to a flat-bottomed trapezoidal profile shape. However, the more numerous strong lines, and consequently the mean LSD profile, come from intermediate longitude to polar regions; therefore an A0 mask represents a good choice for Vega.

The equivalent photospheric profiles contains multiplex information from more than 1100 individual photospheric lines. Typical $S / N$ values of the resulting LSD intensity (Stokes I) profiles are consistently around 1000 per resolved element, following the behaviour pointed out by Donati et al. (1997), and indicating that the convolution model underlying the LSD cannot be trusted above this level of accuracy (this limitation is not valid for the other Stokes profiles). Despite its almost pole-on geometry of $i=7^{\circ}$ (Takeda et al. 2008), the LSD profiles of Vega ( $v \sin i=22 \mathrm{~km} \mathrm{~s}^{-1}$ ) needed to be fitted by rotationally broadened profiles in order to perform an accurate radial velocity determination. The final achieved precision in radial velocity is again $10-15 \mathrm{~m} \mathrm{~s}^{-1}$ for each stellar LSD profile of this data set. The only way to take into account a potential instrumental drift during the night is to work on the difference between stellar and telluric lines, knowing the limitations of the telluric lines as an absolute radial velocity reference.

\section{Search for periodic variations in Vega}

\subsection{Radial velocity variations in Vega}

Due to the one year time gap between the three runs we decided to analyse each run separately. Plotting each radial velocity time series separately, we observed the presence of trends within some nights of less than $100 \mathrm{~m} \mathrm{~s}^{-1} /$ night for the TBL 2008 run, and less than $60 \mathrm{~m} \mathrm{~s}^{-1} /$ night for the CFHT 2009 and TBL 2010 run. Figure 4 shows the gross radial velocity residuals for the 2008 data set, more precisely the difference of stellar to telluric line positions (including a heliocentric rest frame correction).

These slow variations did not appear to be sinusoidal, and we decided to take out these largescale trends by subtracting from the radial velocity curve of each night a fitted 2 nd degree polynomial. In order to check for consistency, we analysed all three couples of detrended and raw radial velocity curves and concluded on the coherence of this approach by detecting the same frequencies in the range above $5 \mathrm{~d}^{-1}$.

Figures 5-7 show the residual radial velocity variations of Vega observed with NARVAL/TBL (2008), ESPaDOnS/CFHT
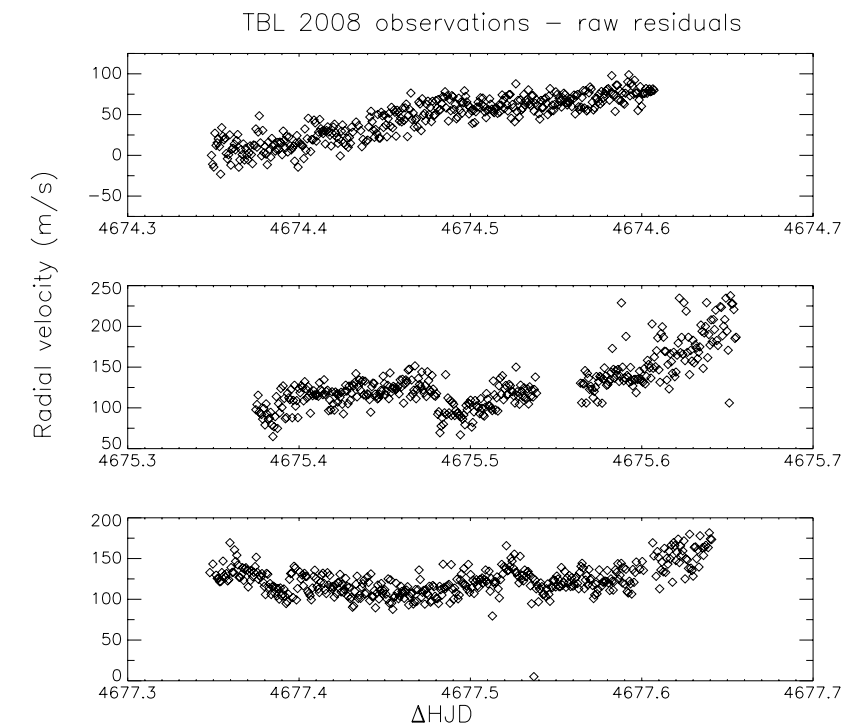

Fig. 4. Residual radial velocity variations of Vega (NARVAL/TBL) 2008, with respect to the first measurement of the run. These data are gross residuals and do still contain a trend during each night. Time is expressed in $\mathrm{HJD}=2450000+\Delta \mathrm{HJD}$.

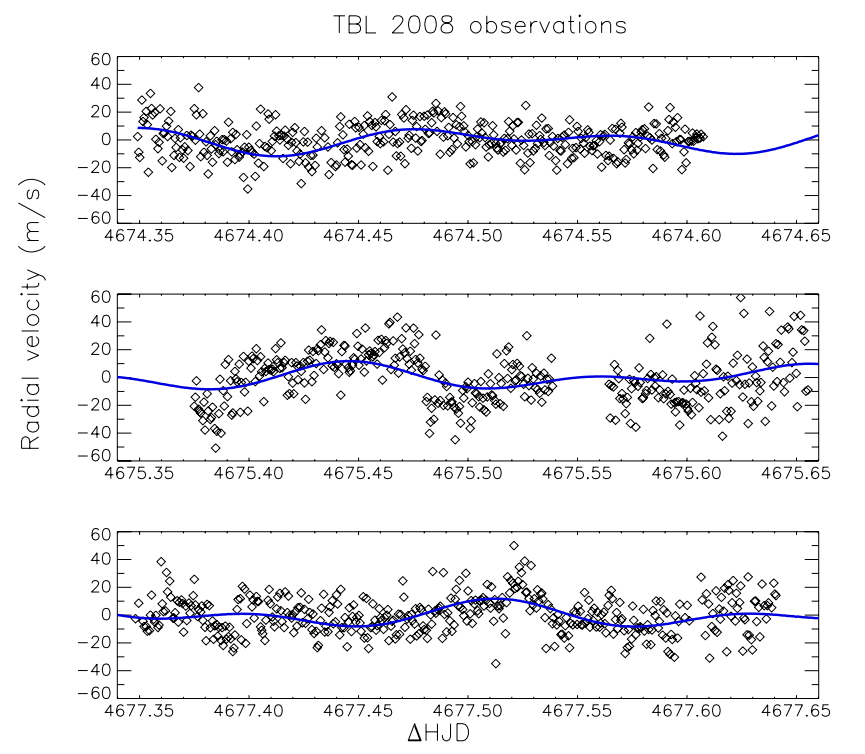

Fig. 5. Residual radial velocity variations of Vega (NARVAL/TBL) 2008. Superimposed (continuous line) is the result of the corresponding frequency analysis (Table 2). Time is expressed in HJD $=2450000+$ $\Delta$ HJD.

(2009) and NARVAL/TBL (2010), respectively. These curves represent the radial velocity variations after the nightly 2 nd degree polynomial detrending. Superimposed are the results of the corresponding frequency analysis (Table 2) with the Period04 package (Lenz \& Breger 2005). This package is based on the discrete Fourier Transform algorithm and multi-sine least-square fits of the data. Interestingly, night 3 of the TBL (2008) data set was the only night in which data were acquired in pure spectroscopic mode; Fig. 5 shows well that the radial velocity curve does in no way depend on the acquisition mode, since variations are of the same order of magnitude and in phase within all three nights. This rules out the polarimetric mode as generator of small radial velocity variations. 

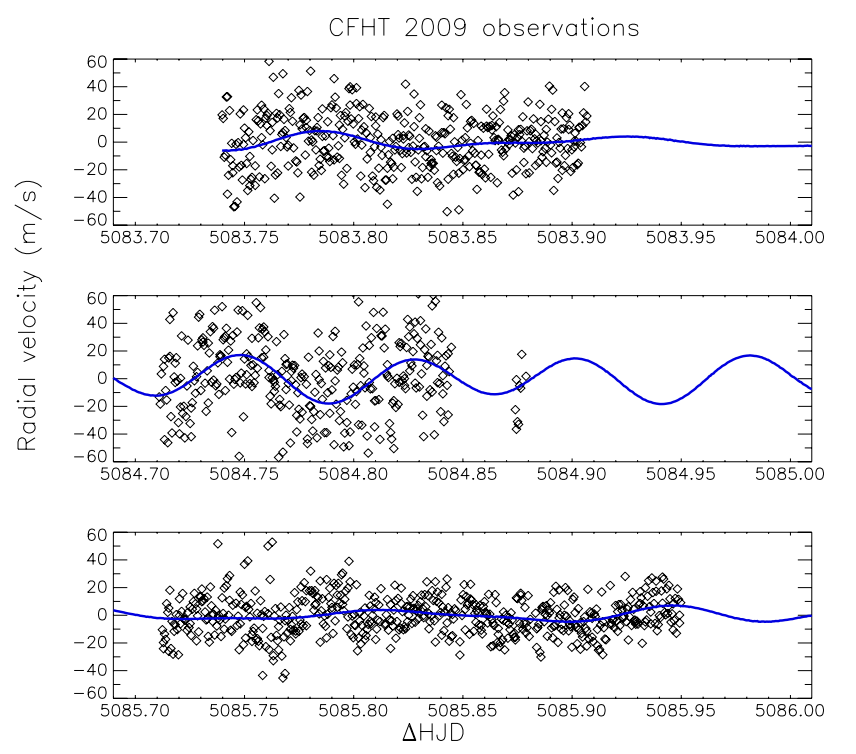

Fig. 6. Residual radial velocity variations of Vega (ESPaDOnS/CFHT) 2009. Superimposed (continuous line) is the result of the corresponding frequency analysis (Table 2). Time is expressed in HJD $=2450000+$ $\Delta$ HJD.

Table 2. Frequencies and amplitudes of the main frequencies.

\begin{tabular}{lcc}
\hline \hline ID & $\begin{array}{c}\text { Freq. } \\
\mathrm{d}^{-1}\end{array}$ & $\begin{array}{c}A \\
\mathrm{~m} \mathrm{~s}^{-1}\end{array}$ \\
\hline$F 1_{2008}$ & 9.19 & 5.8 \\
$F 2_{2008}$ & 5.32 & 5.9 \\
$F 1_{2009}$ & 12.71 & 8.2 \\
$F 2_{2009}$ & 13.25 & 7.8 \\
$F 1_{2010}$ & 5.42 & 4.2 \\
$F 2_{2010}$ & 10.82 & 3.3 \\
\hline
\end{tabular}

Confidence levels have been calculated according tolargescale Breger et al. (1993), who studied empirically the possibility of a peak in the periodogram being a true signal of pulsations with respect to the noise level; this work was refined later by Kuschnig (1997): the significance of a peak in the amplitude periodogram exceeding 4.0 times the mean noise amplitude level after prewhitening of all local frequencies has a $99.9 \%$ probability to be due to stellar pulsations $(99.0 \%$ for a ratio of $3.6,90.0 \%$ for a ratio of 3.2). Figures 8-10 show the results of the Period04 frequency analysis for the three different runs. The two dominant frequencies in the data set of 2008 are $F 1_{2008}=9.19 \mathrm{~d}^{-1}$ $\left(A=5.8 \mathrm{~m} \mathrm{~s}^{-1}\right)$ and $F 2_{2008}=5.32 \mathrm{~d}^{-1}\left(A=5.9 \mathrm{~m} \mathrm{~s}^{-1}\right)$. We stopped the frequency analysis after extraction of the two strongest frequencies, the next potential frequency having a significantly smaller amplitude. In the 2009 data set, the two dominant frequencies are $F 1_{2009}=12.71 \mathrm{~d}^{-1}\left(A=8.2 \mathrm{~m} \mathrm{~s}^{-1}\right)$ and $F 2_{2009}=13.25 \mathrm{~d}^{-1}\left(A=7.8 \mathrm{~m} \mathrm{~s}^{-1}\right)$, the next frequency shows an amplitude of around $3.6 \mathrm{~m} \mathrm{~s}^{-1}$ and was not taken into account. Finally, in the 2010 data set variations are even smaller in amplitude with $F 1_{2010}=5.42 \mathrm{~d}^{-1}\left(A=4.2 \mathrm{~m} \mathrm{~s}^{-1}\right)$. All frequencies of the different data sets are summarised in Table 2; we added a potential second very low amplitude frequency from the 2010 data set for comparison purposes.

\subsection{Line profile variations in Vega}

For almost pole-on objects like Vega, radial velocities are particularly sensitive to zonal modes of low degree $\ell$, if expressed
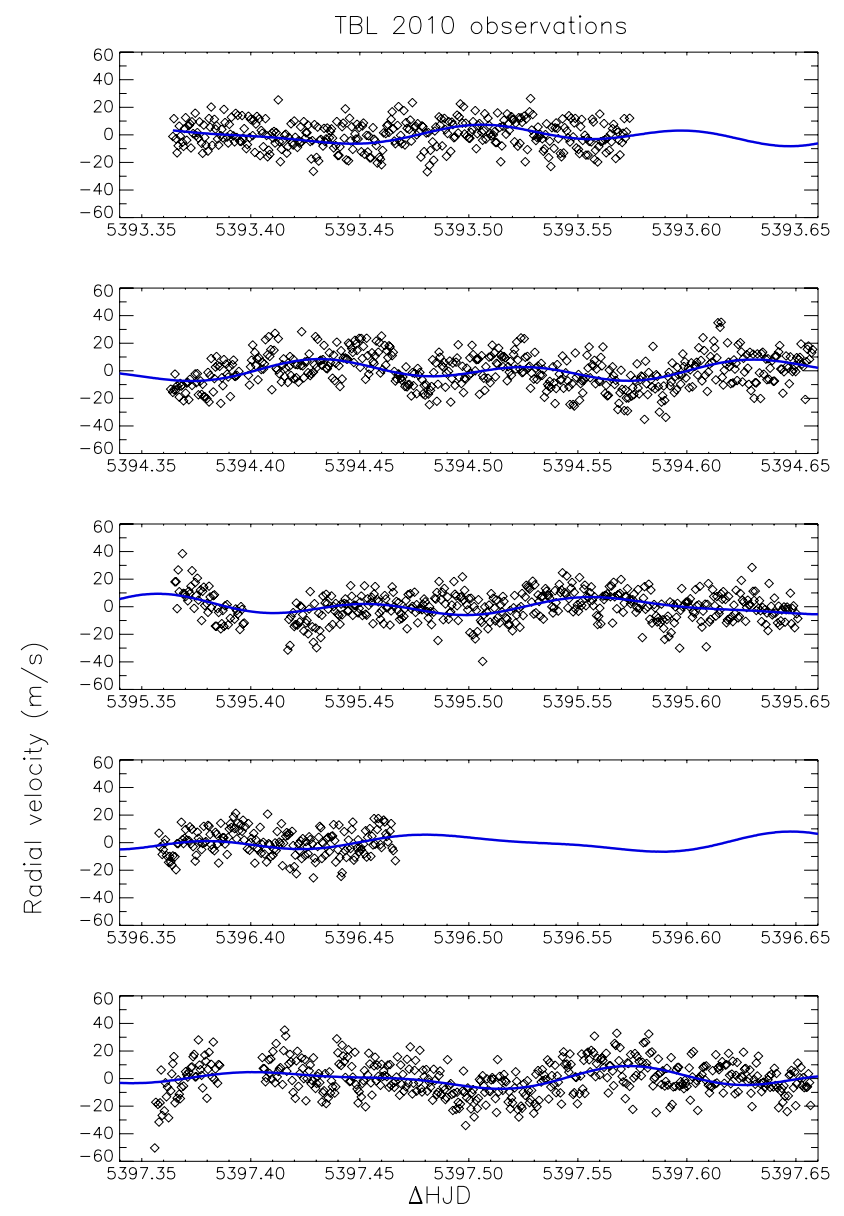

Fig. 7. Residual radial velocity variations of Vega (NARVAL/TBL) 2010. Superimposed (continuous line) is the result of the corresponding frequency analysis (Table 2). Time is expressed in HJD $=2450000+$ $\triangle$ HJD.

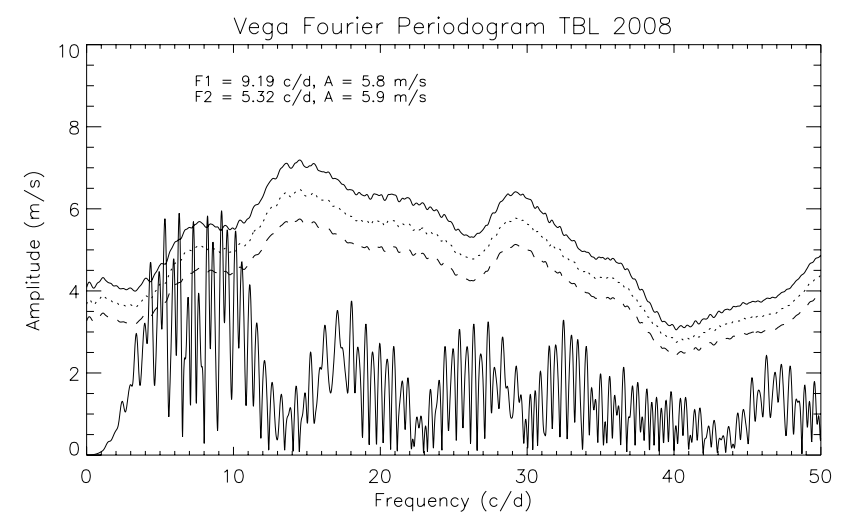

Fig. 8. Result of a Period04 frequency analysis on the NARVAL/TBL data of 2008. Confidence levels are: $99.9 \%$ (cont), $99.0 \%$ (dotted) and $90.0 \%$ level (dashed). These levels were level calculated on a $10 \mathrm{~d}^{-1}$ sliding window after prewhitening with $F 1$ and $F 2$.

in traditional spherical harmonics. We wanted to see if any periodic variation corresponding to a mode of higher degree $\ell$ could be seen in the equivalent photospheric profiles themselves. We therefore analysed the temporal line profile variations of the longest data set (2010) for each individual velocity bin of $1.8 \mathrm{~km} \mathrm{~s}^{-1}$ by computing a 2D (velocity-frequency) LombScargle periodogram (Scargle 1982; Horne \& Baliunas 1986), shown in Fig. 11, after having subtracted the average profile of 


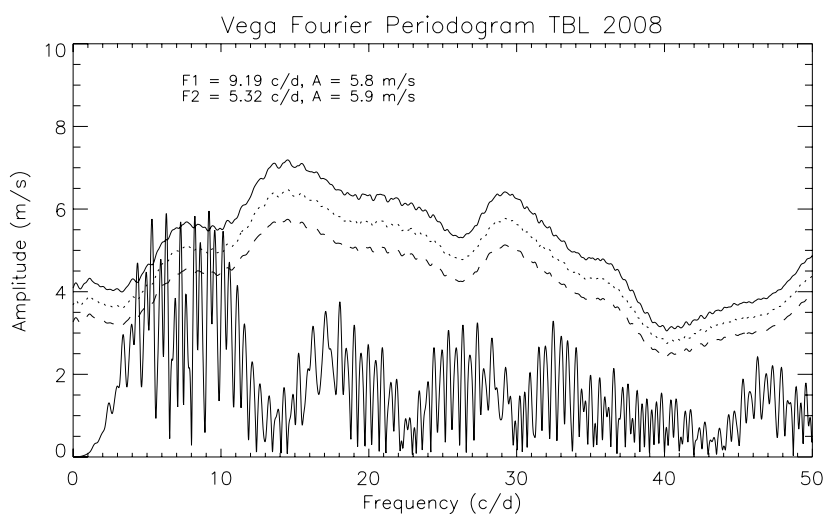

Fig. 9. Result of a Period04 frequency analysis on the ESPaDOnS/CFHT data of 2009. Confidence levels are: $99.9 \%$ (cont), $99.0 \%$ (dotted) and $90.0 \%$ level (dashed). These levels were level calculated on a $10 \mathrm{~d}^{-1}$ sliding window after prewhitening with $F 1$ and $F 2$.

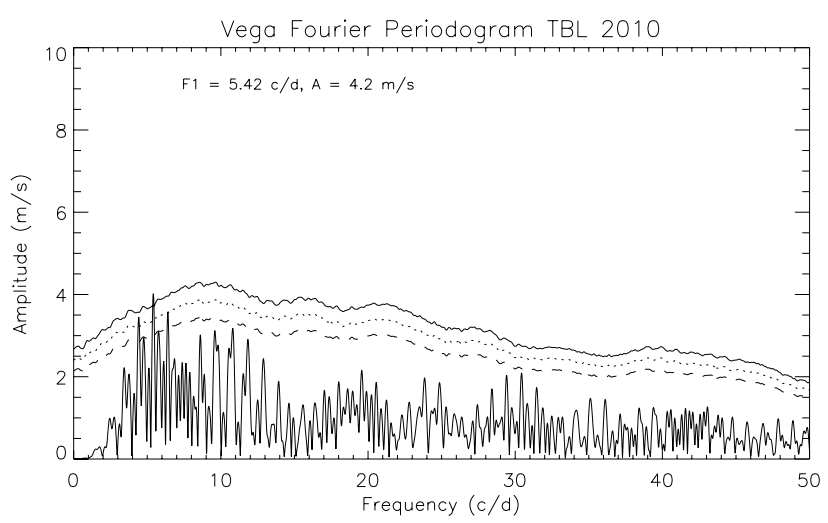

Fig. 10. Result of a Period04 frequency analysis on the NARVAL/TBL data of 2010. Confidence levels are: $99.9 \%$ (cont), $99.0 \%$ (dotted) and $90.0 \%$ level (dashed). These levels were level calculated on a $10 \mathrm{~d}^{-1}$ sliding window after prewhitening with $F 1$.

the run. It should be noted that line profile variations during this run are smaller than $\Delta F / F_{\mathrm{c}}<7 \times 10^{-4}$, where $\Delta F$ and $F_{\mathrm{c}}$ are the profile variations and the (normalized) continuum flux, respectively.

No indication of the presence of any particular frequency within the profile, which was not also present in the continuum, is detected. The weaker signal of the $2 \mathrm{D}$ periodogram within the line profile is due to the square root dependency of the noise, diminishing the absolute noise amplitude within the absorption profile. Figure 12 shows the quadratic sum of the individual Scargle periodograms of all velocity bins within the continuum versus the sum of those within the line. Despite the logarithmic representation, no noticeable difference in the line- versus continuum-Scargle periodogram can be seen.

\section{Discussion and conclusions}

We searched for pulsations in Vega as a near pole-on configuration is particularly favorable for the seismology of rapidly rotating stars.

Equivalent photospheric line profiles were calculated, using the multiplex information of all photospheric spectral lines in the observed wavelength range. In this instrumental configuration, the highest attainable radial velocity calibration was obtained by taking the telluric water vapor line profiles present in the spectrum as absolute wavelength references. A subsequent

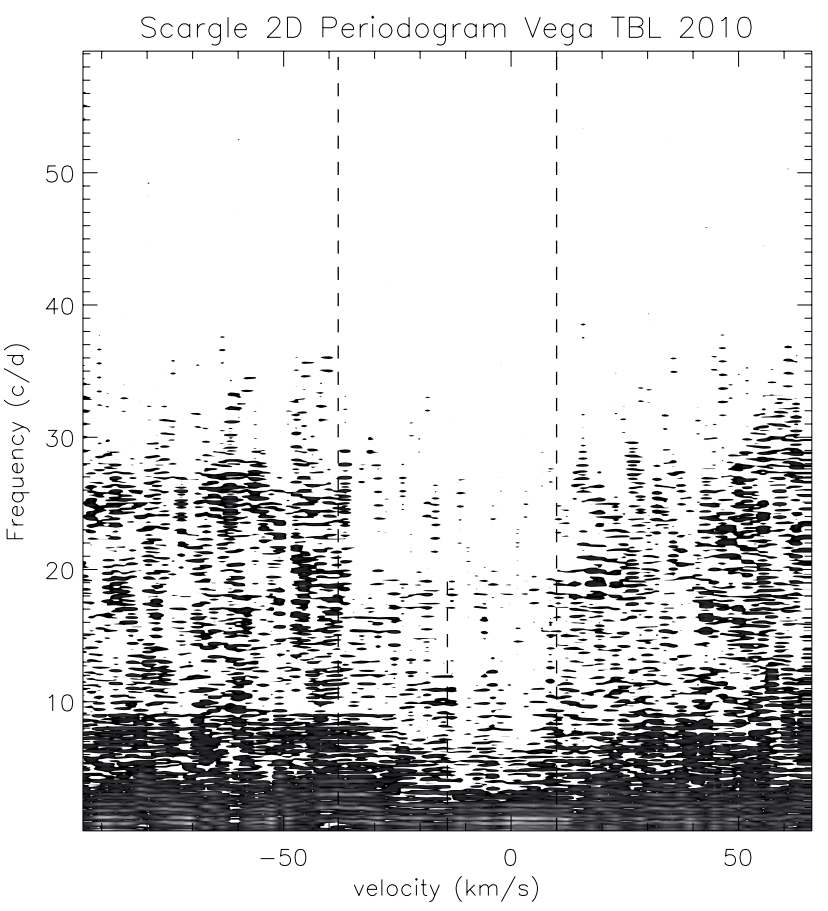

Fig. 11. Two-dimensional Scargle line profile analysis, corresponding to a frequency search for each velocity bin. Data set of 2010 TBL (5 nights). The residual line profile variations have a standard deviation of $0.0007 F / F_{\mathrm{c}}$. Dashed lines indicate the $\pm v \sin$ ilimitations as well as the centroid of the photospheric equivalent profile.

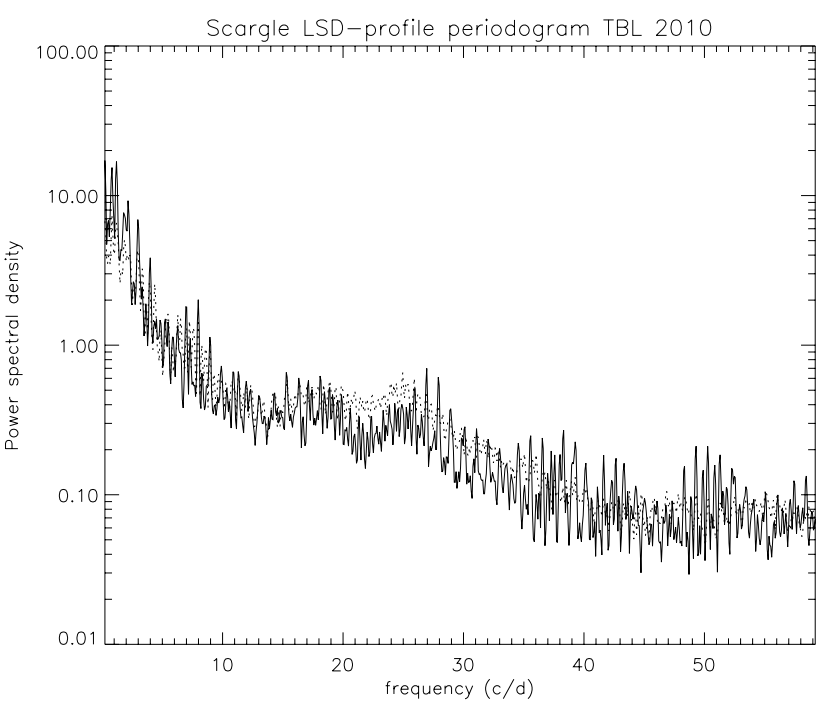

Fig. 12. Equivalent Scargle periodogram for the line (continous) and the continuum (dotted) during the TBL 2010 run, showing that line and continuum have the same frequency pattern, and therefore no indication of periodic line profile variations are detected at this level of precision.

radial velocity analysis with Period04 was performed and low amplitude $\left(A<8 \mathrm{~m} \mathrm{~s}^{-1}\right)$ multi-periodic variations in the residual radial velocity curves were detected, with frequencies ranging roughly from $5-13 \mathrm{~d}^{-1}$, depending on the run. The frequencies detected in this work, if confirmed, are consistent with values occasionally reported in the past, as described in the review by Fernie (1981).

The observed amplitudes of the variations are very small with respect to the intrinsic calibration precision, but taking the telluric water vapor lines of the spectrum as a reference enabled us to reach a precision on the individual radial velocity 
measurement of $10-15 \mathrm{~m} \mathrm{~s}^{-1}$. This auto-calibration of the spectrum should rule out, in principle, any instrumental origin. While the spectroscopic and polarimetric set-up of NARVAL/TBL produces residual radial velocity variations of the same amplitude, and in phase with each other, the configuration of the spectrograph itself can quite certainly be ruled out as an origin. Nevertheless, the similarity of frequencies observed in both runs at TBL (2008 and 2010), quite different to the frequencies observed in 2009 at CFHT, leave us somewhat suspicious concerning a potential, not yet understood, instrumental origin.

The small amplitudes of the detected periodic variations were obtained after detrending the individual nights of each run with 2 nd order polynomials. We must keep in mind that these nightly drifts of the radial velocity were less than $100 \mathrm{~m} \mathrm{~s}^{-1}$ in the 2008 run, and less than $60 \mathrm{~m} \mathrm{~s}^{-1}$ in the CFHT 2009 and TBL 2010 run, and a priori non periodic. No origin could be attributed to these variations. The positive point however was that we were able to detect the periodic signals in both, the raw and the nightly-detrended radial velocity curves.

The intrinsic telluric line variations due to strong, high altitude wind conditions and high airmasses can be of the same order of magnitude as the detected periodicities. These extreme values of intrinsic shifts were certainly not reached, since airmasses during our observations were always small. Moreover, and as an important point, the coherence of the detected periodic signals during 3 or 5 observing nights (depending on the run) and the fact that the variations are not in phase with the beginning of each night tend to rule out the telluric lines as origin of the periodic variations, and strengthen the interpretation as small amplitude stellar pulsations.

The detected frequencies are of the same order as the fundamental mode calculated for Vega. Nevertheless, an excitation mechanism for such modes in this region of the HR Diagram is not known yet.

At this stage we can ascertain that should Vega be confirmed as a pulsator in necessary subsequent high-precision observations with highly stable spectrographs, such as Sophie/OHP, the very small amplitudes observed during the three runs in 2008, 2009 and 2010 show that the star would belong to a category of very "quiet" pulsators. Still, as suggested by Fernie (1981), some rarely occuring, episodic higher amplitude pulsations can not be ruled out. If pulsations were confirmed in the future, Vega would certainly be one of the most interesting stars to monitor in high-precision spectroscopy and photometry.
Another approach to search for rapidly rotating, pulsating and pole-on orientated stars is to search for such candidates within the $\delta$-Scuti instability strip; this work has been recently initiated by our group.

As a conclusion, one thing is certain: Vega has revealed these past years to be a more and more enigmatic star and clearly deserves our further attention!

Acknowledgements. We thank the staffs of TBL and CFHT for their efficient help during these challenging observing runs. F.L. and T.B. acknowledge the support from the French ANR through the SIROCO project GAW acknowledges Discovery Grant support from the Natural Science and Engineering Research Council of Canada (NSERC). We made use of the very helpful Vienna Atomic Line Data base (VALD) in our work.

\section{References}

Aufdenberg, J. P., Mérand, A., Coudé du Foresto, V., et al. 2006, A\&A, 645, 664 Alina, D., Petit, P., Lignières, F., et al. 2011, Proc. of the Conf., Stellar polarimetry: from birth to death, Jun. 27-30, Madison [arXiv: 1107.5639A]

Breger, M., Stich., J., Garrido, R., et al. 1993, A\&A, 271, 482

Caccin, B., Cavallini, F., Ceppatelli, G., Righini, A., \& Sambuco, A. M. 1985, A\&A, 149, 357

Charlton, J. C., \& Meyer, B. S. 1985, PASP, 97, 60

Donati, J.-F., Semel, M., Carter, B. D., Rees, D. E., \& Cameron, A. C. 1997, MNRAS, 291, 658

Fernie, J. D. 1981, PASP, 93, 333

Figueira, P., Pepe, F., Lovis, C., \& Mayor, M. 2010, A\&A, 515, A106

Goraya, P. S., \& Singh, M. 1983, IBVS, 2455, 1

Gray, R. O. 2007, in The Future of Photometric, Spectrophotometric and Polarimetric Standardization, ASP Conf. Ser., 364, 305

Gulliver, A. F., Hill, G., \& Adelman, S. J. 1994, ApJ, 429, L81

Hill, G., Gulliver, A. F., \& Adelman, S. J. 2010, ApJ, 712, 250

Horne, K. D. 1986, PASP, 98, 609

Horne, J. H., \& Baliunas, S. L. 1986, ApJ, 302, 757

Iben, I. 1965, ApJ, 141, 993

Kochukhov, O. P. 2007, Spectrum synthesis for magnetic, chemically stratified stellar atmospheres, Physics of Magnetic Stars. Proceedings of the International Conference, held in the Special Astrophysical Observatory of the Russian AS, August 28-31, 2006, ed. I. I. Romanyuk, \& D. O. Kudryavtsev, 109

Kuschnig, R., Weiss, W. W., Gruber, R., Bely, P. Y., \& Jenkner, H. 1997, A\&A, 328,544

Lenz, P., \& Breger, M. 2005, CoAst, 146, 53

Lignières, F., \& Georgeot, B. 2009, A\&A, 500, 1173

Lignières, F., Petit, P., Böhm, T., \& Aurière, M. 2009, A\&A, 500, L41

Peterson, D. M., Hummel, C. A., Pauls, T. A., et al. 2006, Nature, 440, 896

Petit, P., Lignières, F., Wade, G. A., et al. 2010, A\&A, 523, A41

Scargle, J. D. 1982, ApJ, 263, 835

Takeda, Y., Kawanomoto, S., \& Ohishi, N. 2008, ApJ, 678, 446 